ARTIGO ORIGINAL

ORIGINAL ARTICLE

\title{
Perfil de hospitalizações potencialmente relacionadas à influenza: dados de uma autogestão do Sistema de Saúde Suplementar do Brasil
}

\author{
Hospitalizations potentially attributable to Influenza: data \\ from the Brazilian Private Healthcare System Perspective \\ João Paulo dos Reis Neto', Juliana Martinho Busch', Rafael Rodrigo Araujo², \\ Aline Barbosa², Kelem Chagas², Louis Boiron², Vanessa Teich ${ }^{3}$
}

DOI: 10.21115/JBES.v12.n3.p255-63

\section{Palavras-chave:}

influenza, custos e análise

de custo, hospitalização,

doenças respiratórias, doenças cardiovasculares, saúde suplementar

\section{RESUMO}

Objetivo: Avaliar, com dados de mundo real, hospitalizações por influenza e potencialmente relacionadas à influenza e seus custos associados em uma autogestão do Sistema de Saúde Suplementar do Brasil. Métodos: Estudo retrospectivo na base de dados de uma autogestão, de setembro/2016 a agosto/2019, para avaliar o perfil de hospitalizações por três grupos de doença: influenza/pneumonia, outras doenças respiratórias e doenças cardiovasculares. Foram extraídos números absolutos de hospitalizações para cada grupo, assim como taxas de hospitalização, de re-hospitalização, custos totais e custo médio por paciente. Resultados: Foram registradas 1.047 hospitalizações por influenza/pneumonia, 148 por outras doenças respiratórias e 1.773 por doenças cardiovasculares. A maior taxa de hospitalização ocorreu para doenças cardiovasculares, seguida por influenza/pneumonia. Foram gastos $\mathrm{R} \$ 54,5$ milhões, $\mathrm{R} \$ 32,4$ milhões e $\mathrm{R} \$$ 4,1 milhões com hospitalizações relacionadas a doenças cardiovasculares, influenza/pneumonia e outras doenças respiratórias, respectivamente. O maior custo médio por hospitalização, por paciente, foi observado para influenza/pneumonia (R\$ 30.952), seguido por doenças cardiovasculares (R\$30.740) e outras doenças respiratórias (R\$27.661). Houve um maior número de hospitalizações no grupo com 65 anos ou mais, assim como maiores custos, representando $81,6 \%$ a 92,0\% do custo total de hospitalizações para todas as faixas etárias. Conclusões: Influenza e doenças potencialmente relacionadas a ela, que incluem doenças respiratórias e cardiovasculares, são responsáveis por impactos clínicos e econômicos relevantes, com maiores custos associados às faixas etárias mais altas. Intervenções para minimizar o impacto da influenza, como vacinação, são de extrema relevância para a redução dos custos associados e devem ser consideradas pelos gestores.

\section{ABSTRACT}

Objective: To evaluate, through real-world data, hospitalizations attributed to influenza or potentially attributed to influenza and their costs in a health insurance from the Brazilian Private Healthcare System. Methods: Retrospective study conducted between September 2016 and August 2019 in a health insurance database to assess the hospitalization profile for three disease groups: influenza/pneumonia, other respiratory diseases and cardiovascular diseases. Absolute numbers of

Recebido em: 30/10/2020. Aprovado para publicação em: 15/11/2020.

1. Caixa de Previdência e Assistência dos Servidores da Fundação Nacional de Saúde (Capesesp), Rio de Janeiro, RJ, Brasil. 2. Sanofi Medley Farmacêutica Ltda., São Paulo, SP, Brasil.

3. Consultora em Economia da Saúde, São Paulo, SP, Brasil.

Financiamento: A Sanofi forneceu apoio institucional para a contratação da consultoria responsável pela elaboração do artigo. As análises foram realizadas pela Capesesp sem apoio financeiro. Não houve interferência da Sanofi nas análises de dados realizadas ou na elaboração do artigo.

Instituição onde o trabalho foi executado: CAPESESP - Caixa de Previdência e Assistência dos Servidores da Fundação Nacional de Saúde - Rio de Janeiro, RJ.

Congressos onde o estudo foi apresentado: Não foi apresentado até o momento.

Conflito de interesses: João Paulo dos Reis Neto e Juliana Busch não possuem conflito de interesses a declarar. Vanessa Teich é sócia de uma empresa de consultoria e recebeu honorários para a revisão da análise de dados e elaboração do artigo científico. Autor correspondente: João Paulo dos Reis Neto. Av. Marechal Câmara, 160, 70 andar, Centro, Rio de Janeiro, RJ, Brasil. CEP: 20020-080. Telefone: (21) 2262-6092. E-mail: joaopaulo@capesesp.com.br 
hospitalizations for each group were extracted, as well as hospitalization rate, rehospitalization rate, total costs and average cost per patient. Results: There were 1,047 hospitalizations for influenza/ pneumonia, 148 for other respiratory diseases and 1,773 for cardiovascular diseases. Higher hospitalization rates occurred for cardiovascular disease, followed by influenza/pneumonia. $\mathrm{R} \$ 54.5$ million, R\$32.4 million, and R\$ 4.1 million were spent on hospitalizations attributed to cardiovascular disease, influenza/pneumonia and other respiratory diseases, respectively. The highest average cost per hospitalization, per patient, was observed for influenza/pneumonia (R\$30,952), followed by cardiovascular disease $(R \$ 30,740)$ and other respiratory diseases $(R \$ 27,661)$. A higher number of hospitalizations at older ages was observed, as well as higher costs, representing $81.6 \%$ to $92.0 \%$ of the total hospitalization costs for all age groups. Conclusions: Influenza and diseases potentially attributed to influenza, including respiratory and cardiovascular diseases, are responsible for relevant clinical and economic impacts. Higher hospitalization costs were associated with older age groups. Interventions to minimize the impact of influenza such as vaccination are very relevant to promote a cost reduction and should be considered by health managers.

\section{Introdução}

A influenza é uma doença infecciosa com distribuição global, alta transmissão, tendência à sazonalidade e grande impacto na saúde pública, que pode levar a infecção de vias aéreas inferiores moderada a grave, hospitalização e óbito (World Health Organization, 2012). A real carga associada a essa doença é de difícil mensuração por questões relacionadas ao diagnóstico da infecção, à detecção do vírus influenza como agente etiológico e à indisponibilidade de dados em muitos contextos (GBD, 2018).

A infecção de vias aéreas inferiores representa globalmente a principal causa de mortalidade por doenças infecciosas (GBD, 2018), e aquelas relacionadas à influenza foram responsáveis por 145 mil mortes no mundo, entre todas as idades, em 2017, com taxa de mortalidade geral de 1,9 por 100 mil pessoas. No mesmo ano, foram estimadas 9,5 milhões de hospitalizações e 81,5 milhões de dias de hospitalização por infecções de vias aéreas inferiores atribuíveis à influenza. Para o Brasil, foram estimadas 6 mil mortes atribuíveis à influenza, com taxa de mortalidade geral de 2,8 por 100 mil habitantes e 164 mil hospitalizações nesse período (GBD, 2019).

O impacto da influenza entre idosos se mostra ainda maior. A maioria dos óbitos relacionados à influenza ocorre em pacientes com mais de 70 anos, sendo estimados 71 mil óbitos globalmente e uma taxa de mortalidade de 16,4 mortes por 100 mil habitantes para o ano de 2017 (GBD, 2019). A idade avançada também constitui fator de risco para o desenvolvimento da síndrome respiratória aguda grave (SRAG). No Brasil, entre as semanas epidemiológicas 1 a 32 de 2019, $46,0 \%$ dos óbitos por SRAG relacionada à influenza ocorreram em indivíduos com idade $\geq 60$ anos, sendo esse o principal fator de risco para o óbito (Ministério da Saúde, 2019). A associação mais direta da influenza se dá com o desenvolvimento de pneumonia. A infecção recente por influenza aumenta o risco de pneumonia em aproximadamente 100 vezes (Shrestha et al., 2015). Além das complicações respiratórias, ao longo dos anos, evidências têm demonstrado que a infecção pelo vírus influenza pode ser um fator de risco para a morbimortalidade cardiovascular, insuficiência renal e outras doenças sistêmicas, provavelmente causadas por exacerbação de doenças subjacentes ou comorbidades (Centers for Disease Control and Prevention, 2010; Cohen et al., 2010). De acordo com o Centers for Disease Control and Prevention (CDC), o quadro prévio de influenza constitui um fator de risco para mortes por infarto agudo do miocárdio (IAM), doenças vasculares e diabetes. Porém, por questões relacionadas ao registro conjunto de tais óbitos com influenza, o impacto da influenza pode estar subestimado (Fleming et al., 2005).

No Brasil, o Sistema de Saúde Suplementar atende aproximadamente 47 milhões de pessoas, ou cerca de $24 \%$ da população do país. Sob a perspectiva privada, as hospitalizações representam um relevante impacto financeiro, sendo consideradas o principal direcionador de custos, com 36,9\% do total de custos para o ano de 2018 (R\$ 68,18 bilhões), seguida por exames complementares (18,1\%) e consultas médicas (13,7\%), de acordo com dados da Agência Nacional de Saúde Suplementar (Agência Nacional de Saúde Suplementar, 2018). Na população idosa, a quantidade e o custo de hospitalização são ainda maiores (Agência Nacional de Saúde Suplementar, 2018). Um estudo que avaliou projeções sobre as despesas assistenciais de planos de saúde médico-hospitalares indicou que a parcela com maior crescimento será a de internações, que passará de 57,1\% do total estimado em 2017 para 59,3\% em 2030 (Instituto de Estudos de Saúde Suplementar, 2019).

Quanto às causas de hospitalização no Sistema de Saúde Suplementar, as doenças do aparelho respiratório aparecem em segundo lugar, com 7,1\% do total de internações, atrás apenas das indicações obstétricas (9,4\%). Já as doenças do aparelho circulatório constituem 6,6\% do total de hospitalizações (Agência Nacional de Saúde Suplementar, 2018).

A proporção de beneficiários na última faixa etária entre as autogestões é a maior entre todos os segmentos de operadoras de planos do mercado de saúde suplementar, atingindo, em 2015, seu ápice histórico (30\%) (UNIDAS, 2018).

Em sistemas de saúde com orçamentos limitados e necessidade de utilização racional de recursos, entender o ônus da doença é de grande importância, além de permitir o desenvolvimento de programas de vigilância e intervenção 
(GBD, 2019). Diante desse cenário, o presente estudo teve como objetivo avaliar, com dados de mundo real, hospitalizações relacionadas a influenza, outras doenças respiratórias e doenças cardiovasculares, potencialmente relacionadas à influenza, e seus custos associados em uma autogestão do Sistema de Saúde Suplementar do Brasil.

\section{Métodos}

\section{Desenho do estudo e características gerais da população}

Um estudo não intervencionista retrospectivo avaliou o período de setembro de 2016 a agosto de 2019 da base de dados de uma operadora de saúde para analisar o perfil de hospitalizações por três grupos de doença: influenza/pneumonia, outras doenças respiratórias e doenças cardiovasculares.

Foram considerados elegíveis para inclusão pacientes de qualquer gênero ou faixa etária que apresentassem hospitalização no período de interesse da análise, registrados de acordo com os códigos da Classificação Internacional de Doenças e Problemas Relacionados à Saúde décima edição (CID-10) relacionados a cada um dos três grupos de doença. Para o grupo influenza/pneumonia, foram considerados os códigos relativos a influenza e a pneumonia (J09-J18), uma vez que no dia a dia da prática clínica pode ocorrer descrição apenas da pneumonia, sem associação com o agente etiológico viral ou bacteriano. Para outras doenças respiratórias, J20 e J40-J46 foram considerados. E para doenças cardiovasculares, foram selecionados os códigos 120-125, 150, 164 e G45, com o mesmo objetivo de capturar o maior número de casos relacionados, além de manifestações cardíacas, como, por exemplo, acidente vascular cerebral.

As informações utilizadas para a análise são oriundas da base de dados de uma operadora de saúde pertencente ao Sistema de Saúde Suplementar do Brasil, classificada como autogestão. A Caixa de Previdência e Assistência dos Servidores da Fundação Nacional de Saúde (Capesesp) é uma entidade fechada de previdência complementar patrocinada pela Fundação Nacional de Saúde, Agência Nacional de Vigilância Sanitária, Universidade Federal de Pernambuco, Universidade Federal de Campina Grande, Instituto Brasileiro de Museus, Instituto Federal de Pernambuco e Instituto Federal de Educação, Ciência e Tecnologia da Bahia. Desde 1990, oferece planos de assistência médico-hospitalar que prestam atendimento a cerca de 70 mil beneficiários de todas as faixas etárias (14,11\% de 0 a 19 anos; 59,62\% de 20 a 64 anos; 13,81\% de 65 a 74 anos; 12,46\% com 75 anos ou mais), distribuídos em todo o território nacional.

\section{Extração e análise de dados sobre hospitalizações e custos}

Inicialmente, foram extraídos da base os números absolutos de hospitalizações para cada grupo de patologias de interesse (influenza/pneumonia, outras doenças respiratórias e doenças cardiovasculares) no período do estudo, assim como as taxas de hospitalização, definidas como o número absoluto de hospitalizações dividido pelo número total de beneficiários, expresso em número de hospitalizações por mil usuários.

As análises foram segmentadas em três períodos de tempo (P), conforme descrito a seguir. Para o período 1 (P1), foi considerado o intervalo entre setembro de 2016 e agosto de 2017. O período 2 (P2) foi definido como o espaço de tempo entre setembro de 2017 e agosto de 2018. Já o período 3 (P3) compreendeu a faixa de setembro de 2018 a agosto de 2019.

Taxas de re-hospitalização foram calculadas a partir do número absoluto de pacientes identificados na base pelos CIDs de interesse em cada grupo. O número absoluto de hospitalizações foi dividido pelo número de pacientes com hospitalizações em cada grupo, obtendo-se, então, a taxa de re-hospitalizações para cada grupo de doenças. Os dados foram segmentados por períodos de tempo.

Uma análise adicional sobre hospitalizações foi realizada com a segmentação da coorte do estudo por faixas etárias para cada um dos grupos de doenças e períodos de tempo. As faixas de idade utilizadas foram: 0 a 4 anos; 5 a 9 anos; 10 a 19 anos; 20 a 39 anos; 40 a 59 anos; 60 a 64 anos; 65 a 74 anos; e 75 anos ou mais para influenza/pneumonia e outras doenças respiratórias. Para o grupo de doenças cardiovasculares, foram utilizadas as faixas de 20 a 39 anos, 40 a 59 anos, 60 a 64 anos, 65 a 74 anos, e 75 anos ou mais. Para algumas análises, os dados foram expressos em um subgrupo de pacientes com 65 anos ou mais.

Para a avaliação de custos, o valor total pago para cada grupo de doença nos períodos P1, P2 e P3 foi extraído da base de dados. O custo médio por hospitalização por influenza/pneumonia, outras doenças respiratórias ou doença cardiovascular foi calculado, para cada período de tempo, dividindo-se o valor total obtido pelo número de hospitalizações em cada segmento. Os valores médios de custos correspondentes à média ponderada para os três períodos também foram calculados.

Todos os dados foram analisados de maneira descritiva, considerando medidas de tendência central para variáveis contínuas e medidas de frequência para variáveis categóricas. O programa Microsoft ${ }^{\circ}$ Excel 2018 foi utilizado para as análises.

Uma vez que o estudo foi realizado a partir de análise retrospectiva de banco de dados, sem a identificação dos sujeitos, não houve a necessidade de submissão ao Comitê de Ética em Pesquisa nem, tampouco, de aplicação de termo de consentimento livre e esclarecido, de acordo com a Resolução no 510, de 7 de abril de 2016 (Ministério da Saúde, 2016).

\section{Resultados}

Foram registradas 2.968 hospitalizações para as doenças de interesse no período de setembro de 2016 a agosto de 2019. 
Dessas, 1.047 hospitalizações por influenza/pneumonia, 148 para outras doenças respiratórias e 1.773 para doenças cardiovasculares. As maiores taxas anuais de hospitalização (número de hospitalizações em período de um ano por mil beneficiários) foram observadas para doenças cardiovasculares $(8,31)$, seguidas por influenza/pneumonia $(4,91)$ e outras doenças respiratórias $(0,69)$. Informações segmentadas por grupo de doença e por período de tempo estão apresentadas na Tabela 1.

Para influenza/pneumonia, foram identificados na base 957 pacientes em re-hospitalização. Dividindo-se o número absoluto de hospitalizações por esse valor, a taxa de re-hospitalização foi de 9,40\% no período avaliado. Doenças cardiovasculares apresentaram a maior taxa total de re-hospitalização (17,96\%), e a menor taxa total foi vista entre as outras doenças respiratórias (3,50\%) (Tabela 2).
No período total da análise, foram gastos $\mathrm{R} \$ 54,5 \mathrm{mi}$ Ihões, $\mathrm{R} \$ 32,4$ milhões e $\mathrm{R} \$ 4,1$ milhões com hospitalizações relacionadas a doenças cardiovasculares, influenza/pneumonia e outras doenças respiratórias, respectivamente. No período da análise, o gasto total com hospitalizações foi de $\mathrm{R} \$$ 532.603.755, assim as doenças analisadas representaram $6,1 \%$ do gasto total para influenza/pneumonia, $0,8 \%$ para outras doenças respiratórias e 10,2\% para doenças cardiovasculares. O valor apresentado para o grupo influenza/pneumonia representou 35,6\% do valor total dispensado com todas as hospitalizações analisadas (doenças cardiovasculares, influenza/pneumonia e outras doenças respiratórias). O maior custo médio por paciente foi observado para influenza/pneumonia ( $R \$ 30.952)$, seguido por doenças cardiovasculares ( $R \$ 30.740$ ) e outras doenças respiratórias (R\$27.661). As análises segmentadas por períodos de tempo estão apresentadas na Tabela 3.

Tabela 1. Número absoluto de hospitalizações e taxa de hospitalização para influenza, outras doenças respiratórias e doenças cardiovasculares, segmentados por períodos de tempo. CAPESESP, SET/2016-AGO/2019.

\begin{tabular}{|c|c|c|c|c|c|c|c|c|}
\hline Grupo & $\begin{array}{c}\text { Hospitalizações } \\
\text { (P1), N }\end{array}$ & $\begin{array}{c}\text { Taxa de } \\
\text { hospitalização } \\
(\mathrm{P} 1) / 1.000\end{array}$ & $\begin{array}{l}\text { Hospitalizações } \\
\text { (P2), N }\end{array}$ & $\begin{array}{c}\text { Taxa de } \\
\text { hospitalização } \\
(\mathrm{P} 2) / 1.000\end{array}$ & $\begin{array}{l}\text { Hospitalizações } \\
\text { (P3), N }\end{array}$ & $\begin{array}{c}\text { Taxa de } \\
\text { hospitalização } \\
(\mathrm{P} 3) / 1.000\end{array}$ & $\begin{array}{c}\text { Total de } \\
\text { hospitalizações, } \\
\text { N }\end{array}$ & $\begin{array}{c}\text { Taxa anual de } \\
\text { hospitalização* }\end{array}$ \\
\hline Influenza / Pneumonia & 302 & 3,17 & 320 & 4,47 & 425 & 6,98 & 1.047 & 4,91 \\
\hline Outras doenças respiratórias & 38 & 0,40 & 53 & 0,74 & 57 & 0,94 & 148 & 0,69 \\
\hline Doenças cardiovasculares & 604 & 6,34 & 535 & 7,47 & 634 & 10,41 & 1.773 & 8,31 \\
\hline Total & 944 & 9,90 & 908 & 12,68 & 1.116 & 18,33 & 2.968 & 13,92 \\
\hline
\end{tabular}

N: número absoluto; P1: período entre setembro de 2016 e agosto de 2017; P2: período entre setembro de 2017 e agosto de 2018; P3: período entre setembro de 2018 e agosto de 2019.

*Taxa anual de hospitalização calculada como a média ponderada dos períodos P1, P2 e P3.

Tabela 2. Número absoluto de pacientes identificados na base para cada grupo e taxa de re-hospitalização para influenza, outras doenças respiratórias e doenças cardiovasculares, segmentados por períodos de tempo. CAPESESP, SET/2016-AGO/2019.

\begin{tabular}{|c|c|c|c|c|c|c|c|c|}
\hline Grupo & $\begin{array}{c}\text { Pacientes } \\
\text { identificados na } \\
\text { base }(P 1), N\end{array}$ & $\begin{array}{c}\text { Taxa de } \\
\text { re-hospitalização } \\
\text { (P1), \% }\end{array}$ & $\begin{array}{c}\text { Pacientes } \\
\text { identificados na } \\
\text { base }(\mathrm{P} 2), \mathrm{N}\end{array}$ & $\begin{array}{c}\text { Taxa de } \\
\text { re-hospitalização } \\
\text { (P2), \% }\end{array}$ & $\begin{array}{c}\text { Pacientes } \\
\text { identificados na } \\
\text { base (P3), N }\end{array}$ & $\begin{array}{c}\text { Taxa de } \\
\text { re-hospitalização } \\
\text { (P3), \% }\end{array}$ & $\begin{array}{c}\text { Total de } \\
\text { pacientes } \\
\mathrm{N}\end{array}$ & $\begin{array}{c}\text { Taxa total de } \\
\text { re-hospitalização, } \\
\%^{*}\end{array}$ \\
\hline Influenza / Pneumonia & 278 & 8,6 & 296 & 8,1 & 383 & 11,0 & 957 & 9,40 \\
\hline Outras doenças respiratórias & 36 & 5,6 & 51 & 3,9 & 56 & 1,8 & 143 & 3,50 \\
\hline Doenças cardiovasculares & 473 & 27,7 & 475 & 12,6 & 555 & 14,2 & 1.503 & 17,96 \\
\hline Total & 787 & 19,9 & 822 & 10,5 & 994 & 12,3 & 2.603 & 14,02 \\
\hline
\end{tabular}

N: número absoluto; P1: período entre setembro de 2016 e agosto de 2017; P2: período entre setembro de 2017 e agosto de 2018; P3: período entre setembro de 2018 e agosto de 2019.

*Taxa total de re-hospitalização calculada como a média ponderada dos períodos P1, P2 e P3.

Tabela 3. Valor total e custo médio por paciente para influenza, outras doenças respiratórias e doenças cardiovasculares, segmentados por períodos de tempo. CAPESESP, SET/2016-AGO/2019.

\begin{tabular}{|c|c|c|c|c|c|c|c|c|}
\hline Grupo & $\begin{array}{l}\text { Valor total } \\
\text { (P1), R\$ }\end{array}$ & $\begin{array}{c}\text { Custo } \\
\text { médio por } \\
\text { hospitalização } \\
\text { (P1), R\$ }\end{array}$ & $\begin{array}{l}\text { Valor total } \\
\text { (P2), R\$ }\end{array}$ & $\begin{array}{c}\text { Custo } \\
\text { médio por } \\
\text { hospitalização } \\
\text { (P2), R\$ }\end{array}$ & $\begin{array}{l}\text { Valor total } \\
\text { (P3), R\$ }\end{array}$ & $\begin{array}{c}\text { Custo } \\
\text { médio por } \\
\text { hospitalização } \\
\text { (P3), R\$ }\end{array}$ & Valor total, R\$ & $\begin{array}{l}\text { Custo médio por } \\
\text { hospitalização, R\$ }\end{array}$ \\
\hline Influenza / Pneumonia & 7.359 .087 & 24.368 & 11.314 .056 & 35.356 & 13.733 .352 & 32.314 & 32.406 .494 & 30.952 \\
\hline Outras doenças respiratórias & 754.236 & 19.848 & 1.805 .948 & 34.074 & 1.533 .657 & 26.906 & 4.093 .841 & 27.661 \\
\hline Doenças cardiovasculares & 18.745 .373 & 31.035 & 17.318.119 & 32.370 & 18.439 .065 & 29.084 & 54.502 .557 & 30.740 \\
\hline Total & 26.858 .696 & 28.452 & 30.438 .122 & 33.522 & 33.706 .074 & 30.203 & 91.002 .892 & 30.661 \\
\hline
\end{tabular}

P1: período entre setembro de 2016 e agosto de 2017; P2: período entre setembro de 2017 e agosto de 2018; P3: período entre setembro de 2018 e agosto de 2019. 
Em toda a base de dados, sem a utilização de filtro por CID, foram localizadas 16.698 hospitalizações, sendo 10.338 com algum CID registrado e 6.360 sem CID registrado, ou seja, apenas $62 \%$ das hospitalizações possuíam registro de CID. Ao se aplicar esse percentual ao número de hospitalizações identificadas por CID (Tabela 1), foi possível estimar um número ainda maior de hospitalizações para os três grupos de doenças de interesse, que passou de 2.968 para 4.794 no período de três anos de análise (Tabela 4).

A análise segmentada por faixas etárias mostrou mais hospitalizações em idades avançadas. Entre aqueles do grupo influenza/pneumonia (Tabela 5), as maiores taxas de hospitalização foram vistas no grupo com 75 anos ou mais, atingindo uma taxa anual de 25,25 por mil pacientes. Foi realizada uma subanálise para os pacientes com 65 anos ou mais de idade, em que a taxa anual de hospitalização foi de 14,54 por mil pacientes. A população com 65 anos ou mais, que representa $26,3 \%$ da carteira, mostrou um custo total de R\$ 28.760.457, somando-se os três períodos de análise, correspondendo a $88,7 \%$ do valor total para todas as faixas etárias. O custo médio ponderado para a hospitalização de um paciente com 65 anos ou mais foi de $\mathrm{R} \$ 35.289$.

Para o grupo de outras doenças respiratórias (Tabela 6), a subanálise para pacientes com 65 anos ou mais de idade mostrou uma taxa anual de hospitalização igual a 1,82 por mil pacientes. Entre os valores totais gastos com todas as faixas etárias, pacientes com 65 anos ou mais representaram 92,0\% do total, com um custo médio por hospitalização de $\mathrm{R} \$ 36.934$

Considerando a amplitude de idade compreendida para o grupo de doenças cardiovasculares (20 anos ou mais), a taxa anual de hospitalização atingiu 28,75 por mil pacientes para aqueles com 75 anos ou mais. A mesma taxa para aqueles com 65 anos ou mais foi de 22,40 por mil pacientes (Tabela 7), com custos responsáveis por 81,4\% do total.

\section{Discussão}

Apesar dos avanços vistos nos últimos anos sobre as análises do impacto da influenza, sua real carga ainda não é totalmente esclarecida, uma vez que envolve fatores como subdiagnóstico, subregistro e associação com outras patologias, deixando essa etiologia mascarada em muitos casos. Considerando a necessidade de maior compreensão desse impacto em nível local, uma análise de base de dados de uma autogestão foi conduzida com o objetivo de fornecer dados de mundo real sobre o perfil de hospitalizações e seus custos associados. Uma vez que a infecção pelo vírus influenza é considerada um fator de risco para doença cardiovascular e que esse grupo constitui uma causa relevante de hospitalização na saúde suplementar, três grupos de doenças foram então definidos para a análise, sendo eles influenza/pneumonia, outras doenças respiratórias e doenças cardiovasculares.

Em indivíduos saudáveis, apesar do comprometimento infeccioso sistêmico associado à influenza, esta pode apresentar evolução benigna. Porém, em determinados grupos, como aqueles com idade abaixo de 2 anos ou acima de 60 anos, e pacientes com doenças crônicas, o impacto da doença na morbimortalidade ocorre de forma muito mais intensa (Daufenbach et al., 2009). Em concordância com esses dados, no presente estudo, a análise segmentada por faixas etárias mostrou maior número de hospitalização em idades mais avançadas para os três grupos de doenças avaliadas. Os custos entre idosos de 65 anos ou mais acompanham a mesma tendência, sendo responsáveis por valores que representaram de $81,6 \%$ a $92,0 \%$ do valor total de hospitalizações para todas as faixas etárias, ao passo que representam, em termos de número de pacientes, apenas $26,3 \%$ da carteira. Adicionalmente, em uma série temporal que avaliou a mortalidade por doenças respiratórias relacionadas a influenza em pessoas com 60 anos de idade ou mais, no Brasil, observou-se tendência crescente nas taxas de mortalidade com o aumento da idade da população (Campagna et al., 2009). A participação da população idosa na saúde suplementar vem aumentando com o passar dos anos e, em avaliação por tipo de operadora, o envelhecimento populacional mostrou aumento ainda maior na modalidade de autogestão (Agência Nacional de Saúde Suplementar, 2016).

No cenário internacional, um estudo de vigilância com base populacional nos Estados Unidos cita que rotineiramente há subregistro de casos de influenza, com necessidade de utilização de um fator multiplicador para visualizar a real dimensão do impacto da doença (Reed et al., 2015).

Tabela 4. Projeção do total de internações após ajuste das internações sem CID registrado.

\begin{tabular}{|c|c|c|c|c|c|c|c|c|}
\hline & $N(P 1)$ & Taxa (P1) & $N(P 2)$ & Taxa (P2) & $N(P 3)$ & Taxa (P3) & $\begin{array}{l}\text { Total no } \\
\text { período }\end{array}$ & $\begin{array}{c}\text { Taxa (Total no } \\
\text { período) }\end{array}$ \\
\hline Internações com CID registrado & 3.091 & -- & 3.105 & --- & 4.142 & --- & 10.338 & --- \\
\hline Internações sem CID registrado & 1.865 & --- & 3.191 & --- & 1.304 & --- & 6.360 & --- \\
\hline Total de internações & 4.956 & -- & 6.296 & --- & 5.446 & --- & 16.698 & --- \\
\hline Internações com CID registrado (\%) & 62 & --- & 49 & --- & 76 & --- & 62 & --- \\
\hline \multicolumn{9}{|l|}{ Estimativa do total de internações } \\
\hline nos CIDs analisados & 1.514 & 15,88 & 1.841 & 25,72 & 1.467 & 24,09 & 4.794 & 67,45 \\
\hline
\end{tabular}




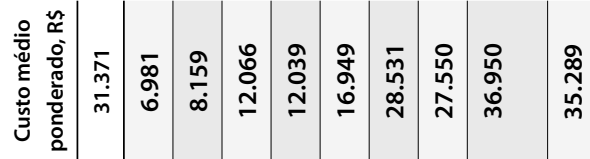

흥용

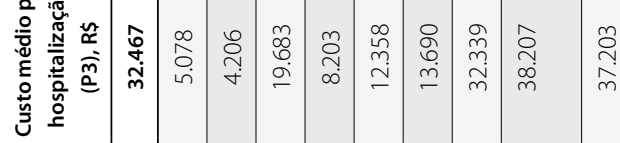

㶾

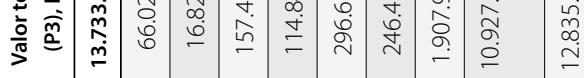

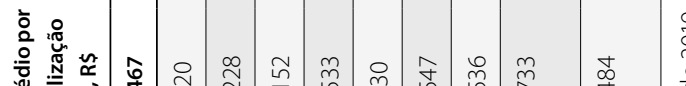

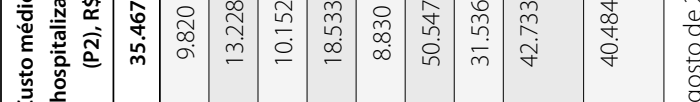

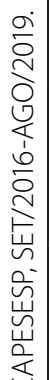

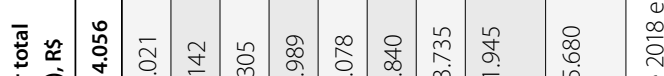

$\stackrel{\circ}{\sigma}$

定

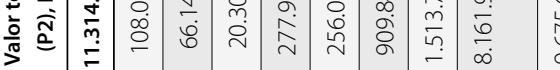

(5)

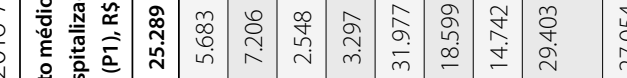

政

$\stackrel{\circ}{\stackrel{\circ}{\varepsilon}}$

$\frac{0}{0}$

일

을

.

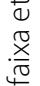

高

$\frac{1}{0}$

है

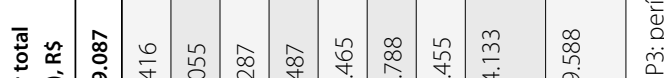

产

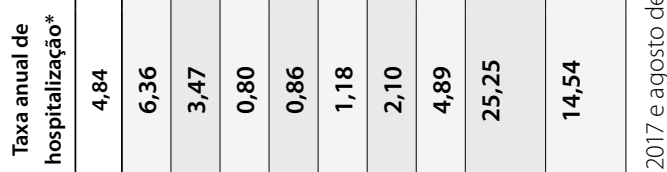

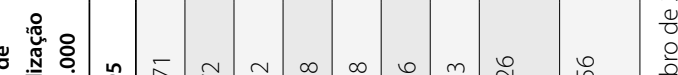

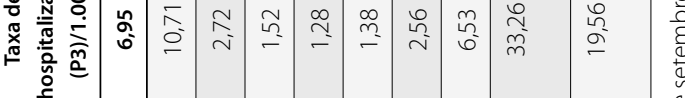

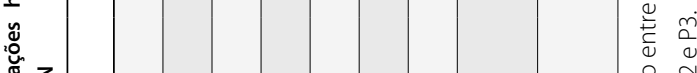

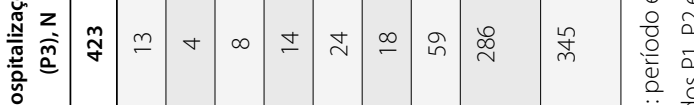

这

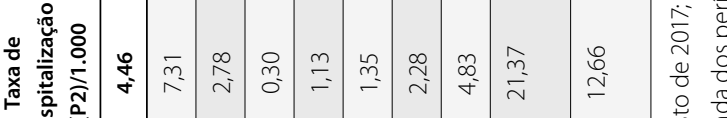

๗

:

है

$\stackrel{\frac{\pi}{2}}{\frac{\pi}{4}}$

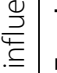

(5)

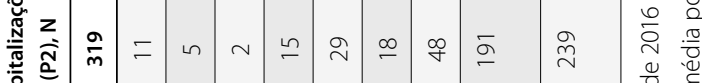
옹

)

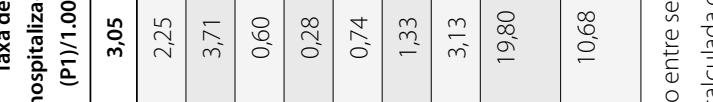

聯

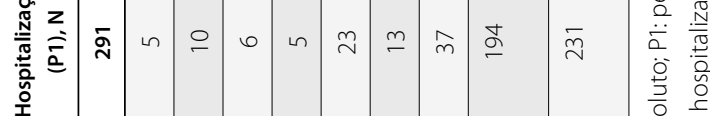

苞

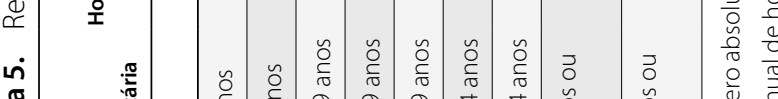

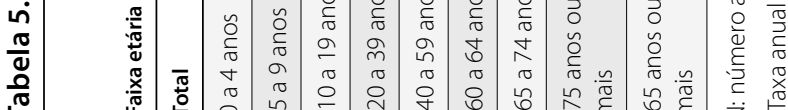

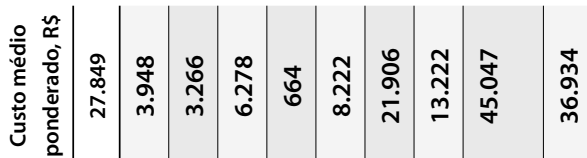

ㅎ․ㅇํㅇ

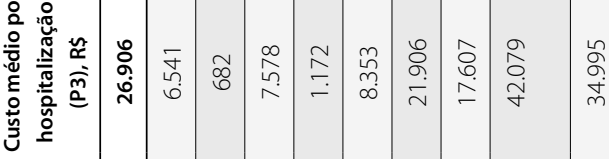

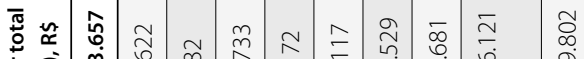

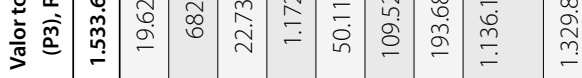

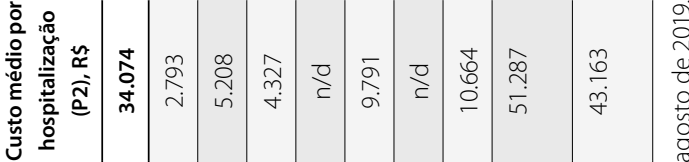

ळे

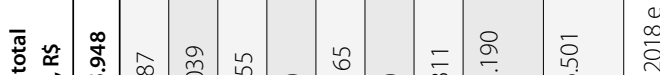

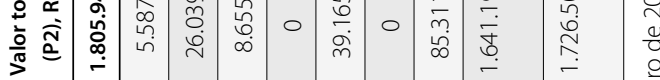

离

है

$\frac{8}{0}$

กั

वे

竞

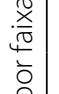

$\frac{2}{0}$

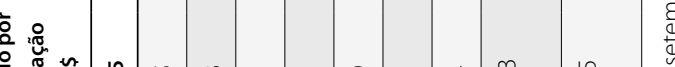

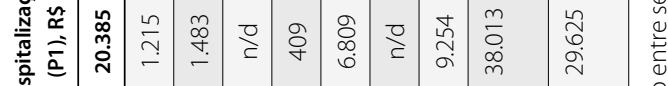

旁

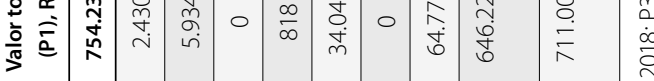

ㄴ. 웅

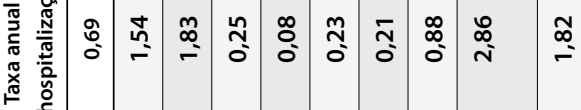

:

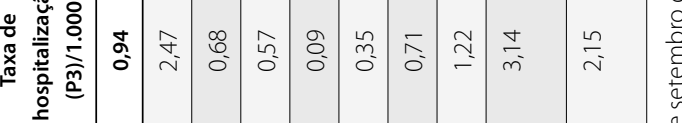

䢰

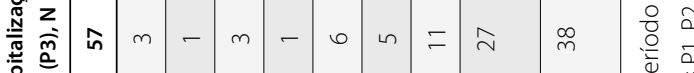
薃

造

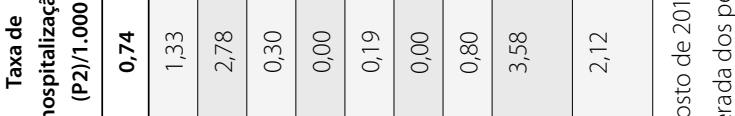

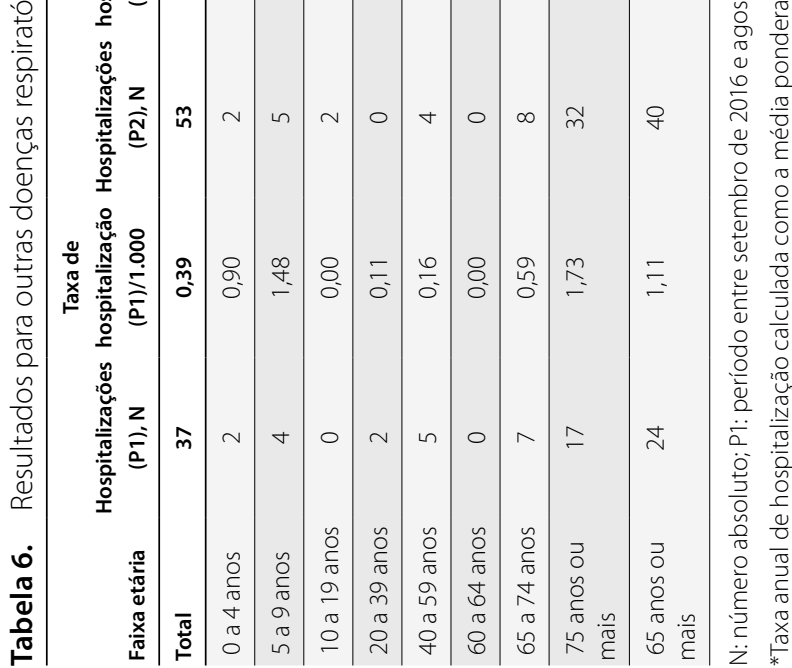




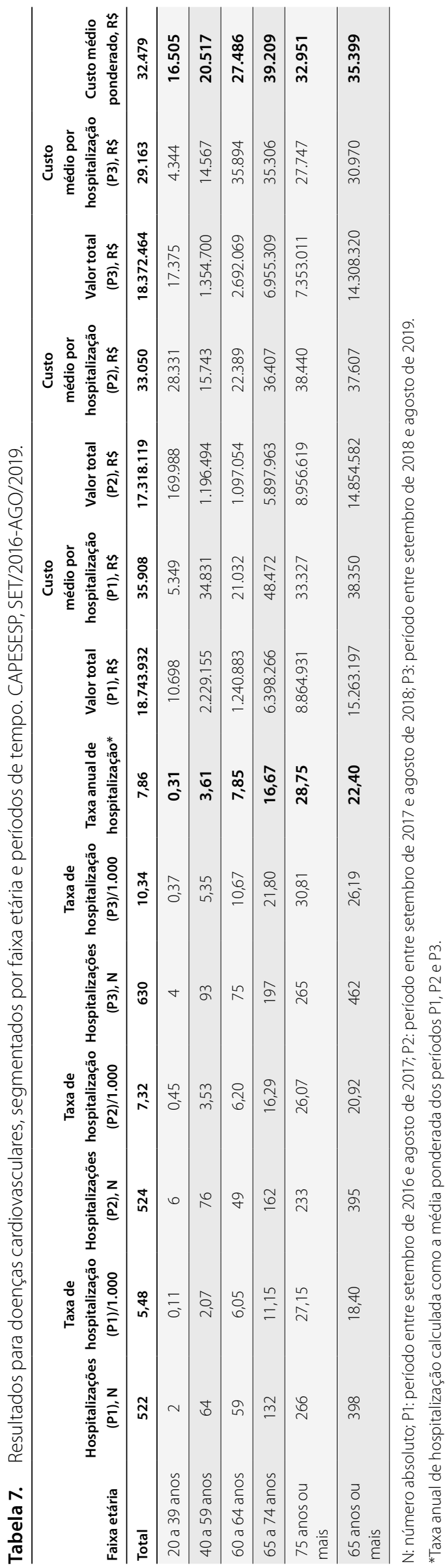

Problemas relacionados ao subregistro não só da influenza, mas de qualquer patologia em casos de hospitalização, são frequentes. Na análise desenvolvida e aqui apresentada, apenas 62\% das hospitalizações possuíam registro de CID-10. Além do subregistro, a análise dos dados foi capaz de identificar taxas de re-hospitalização para o grupo influenza/pneumonia, variando de $8,1 \%$ a $11,0 \%$ nos períodos avaliados, com maiores taxas entre as doenças cardiovasculares, atingindo até $27,7 \%$. A realização do teste confirmatório para influenza não é rotineira, nem mesmo nos casos de doença respiratória em período sazonal. Dessa forma, torna-se difícil estimar com precisão a associação das internações com influenza, o que justifica a seleção dos CID-10 deste estudo, bem descrita pela literatura mundial (Reed et al., 2015).

Apesar de representar $35,6 \%$ do valor total dispensado com todas as hospitalizações analisadas, o grupo influenza/ pneumonia apresentou o maior custo médio por paciente ( $R \$ 30.952$ ), seguido por doenças cardiovasculares ( $R \$ 30.740$ ) e outras doenças respiratórias (R\$ 27.661). Em pesquisa nacional promovida pela União Nacional das Instituições de Autogestão em Saúde, o custo médio de hospitalização para a faixa etária de 59 anos ou mais foi de $\mathrm{R} \$ 27.046$, próximo ao valor encontrado nesta análise (Agência Nacional de Saúde Suplementar, 2018). A pesquisa nacional mostra que o custo médio da internação para essa faixa etária é quatro vezes maior do que o custo para aqueles até 18 anos (R\$ 6.619) (Agência Nacional de Saúde Suplementar, 2018). Uma revisão da literatura avaliou estudos que estimaram o custo associado à influenza sazonal. Os custos por admissão foram mais altos nos EUA (US\$ 11.096 a 83.216 por hospitalização) e mais baixos na Tailândia (US\$ 304 a 340 por admissão hospitalar). Os custos de hospitalização na França, Noruega e Austrália foram consistentes (US\$ 4.154, US\$ 4.717 e US\$ 4.516, respectivamente) e mais elevados do que no Japão (US\$1.333) e Hong Kong (US\$ 529) (Peasah et al., 2013).

A infecção por influenza pode multiplicar por dois ou mais o risco de desenvolvimento de pneumonia por Streptococcus pneumoniae. Caracterizada como uma doença sistêmica, a influenza pode levar não só à pneumonia, mas também exacerbar doenças crônicas subjacentes, culminando em complicações cardiovasculares, renais, metabólicas e neurológicas, especialmente nos extremos de idade e em imunocomprometidos (Bricks et al., 2015). Nesse cenário, o desenvolvimento de programas de vigilância e intervenções capazes de minimizar casos de influenza e seus impactos são muito relevantes em termos de saúde pública, para todos os provedores envolvidos, incluindo a saúde suplementar brasileira, a fim de melhorar desfechos clínicos e minimizar custos com hospitalizações.

As campanhas de vacinação contra gripe tiveram início no Brasil em 1999. Inicialmente, a vacina foi disponibilizada para os indivíduos com idade $\geq 65$ anos e, a partir do ano 2000, 
para a população com idade $\geq 60$ anos (Bacurau et al., 2019). Como estratégia para redução das internações, complicações e óbitos associados à infecção por influenza, o Ministério da Saúde recomenda uma dose anual da vacina para os grupos considerados de risco, incluindo idosos e portadores de doenças crônicas (Ministério da Saúde, 2017). A dose anual da vacina também é recomendada pelas diretrizes da American Heart Association e do American College of Cardiology para pacientes com doença aterosclerótica em geral (Smith et al., 2011). A Sociedade Brasileira de Cardiologia também indica a vacina contra influenza para pacientes com insuficiência cardíaca (Bocchi et al., 2009). Apesar da disponibilização gratuita da vacina pelo Ministério da Saúde, a vacinação não tem atingido a meta de cobertura considerada adequada para todos grupos-alvo (Domingues \& Oliveira, 2012).

Dados da literatura mostram que pode haver imprecisão na avaliação da taxa de cobertura vacinal. No Brasil, a avaliação sistemática das doses administradas nas unidades de saúde estimou a cobertura vacinal em 89,1\% em 2015 e 97,1\% em 2016. Porém, a metodologia utilizada foi considerada limitada por premissas imprecisas, levando a superestimativas da cobertura vacinal (Sato et al., 2020). Em um estudo transversal envolvendo 5.221 indivíduos com 60 anos ou mais, a cobertura da vacinação contra influenza foi de 73,0\% (Sato et al., 2020), e a meta atual para esse grupo é de $90 \%$. Um inquérito domiciliar ( $N=365)$ da população urbana maior de 60 anos em Botucatu, São Paulo, registrou cobertura vacinal de 63,2\% (IC 95\%: 58,3-68,2) para o ano de 2002. Foi observado menor percentual de vacinados entre os idosos na faixa etária de 60 a 64 anos (40,9\%). Com exceção dos hipertensos, a população que referiu ser portadora de doenças crônicas como diabetes mellitus, doença cardíaca e/ou doença pulmonar apresentou cobertura vacinal semelhante à que não referiu tais doenças, não atingindo níveis adequados conforme esperado para esse grupo de risco (Donalisio et al., 2006). Em um estudo transversal de base populacional que incluiu 1.517 idosos ( $\geq 60$ anos) em Campinas, no período de 2008 a 2009 , apenas $62,6 \%$ da coorte autorreportou ser vacinada. Exceto por diabetes, a cobertura vacinal não foi maior, conforme esperado, para populações com doenças crônicas. As razões mais citadas para a não adesão foram: não considerar a vacina necessária (46,5\%), crença de que a vacina provoca reação (36,7\%), falta de orientação $(3,6 \%)$ e dificuldade de acesso (2,2\%) (Francisco et al., 2011). Já na cidade de São Paulo, entre 1.399 idosos, a realização da vacina autorreferida foi de 73,8\% para 2006, associada à idade mais elevada, à presença de doenças crônicas e ao atendimento à saúde no ano anterior (Moura et al., 2015).

No que tange à avaliação da vacina como potencial redutor da morbidade associada à influenza, no Brasil, um estudo ecológico entre 1992 e 2006 descreveu o coeficiente de morbidade hospitalar por causas relacionadas à influenza na população brasileira $\geq 60$ anos de idade. Ao comparar o coeficiente médio de morbidade hospitalar dos períodos anterior (1992-1998) e posterior (1999-2006) à introdução da vacinação contra influenza no país, percebeu-se redução do coeficiente neste último período, sobretudo nas regiões Sul, Centro-Oeste e Sudeste. O estudo ressalta que a diminuição dos coeficientes após 1999 pode estar relacionada às campanhas de vacinação (Daufenbach et al., 2009).

Apesar da relevância dos dados apresentados para auxílio na compreensão do impacto da influenza na saúde suplementar, a utilização de CID-10 relacionados a pneumonia como critério de inclusão para o grupo influenza pode ser considerada como uma limitação. Problemas relacionados ao diagnóstico e ao registro correto para casos de influenza podem justificar tal critério de elegibilidade. Da mesma forma, outros autores também se utilizaram de códigos de doença, sem definição de etiologia, para o desenvolvimento de seus estudos. Daufenbach e cols., Campagna e cols. e Daufenbach e cols. definiram grupos como pneumonia e influenza, bronquite crônica e não especificada, e obstrução crônica de vias respiratórias como causas relacionadas à influenza (Daufenbach et al., 2009; Campagna et al., 2009; Daufenbach et al., 2014). Outras potenciais limitações dizem respeito ao correto preenchimento dos dados contidos na base, assim como à qualidade deles.

\section{Conclusões}

A influenza e as doenças potencialmente relacionadas a ela, que incluem doenças respiratórias, em particular pneumonia, e doenças cardiovasculares, são responsáveis por impactos clínicos e econômicos relevantes. Os maiores custos ocasionados por hospitalizações relacionadas à influenza/ pneumonia foram associados às faixas etárias mais altas no contexto de uma autogestão, no Sistema de Saúde Suplementar. Intervenções capazes de minimizar o impacto da influenza, como a ampla cobertura vacinal e a disponibilização de vacinas mais efetivas, são de extrema relevância para a saúde pública e para o contexto da saúde privada, no Brasil.

\section{Referências bibliográficas}

Agência Nacional de Saúde Suplementar. Dados assistenciais do setor por semestre [Internet]. 2018. Available from: http://www.ans.gov.br/perfildo-setor/dados-e-indicadores-do-setor. Accessed on: Dec 17, 2019.

Agência Nacional de Saúde Suplementar. Idoso na saúde suplementar: uma urgência para a saúde da sociedade e para a sustentabilidade do setor [Internet]. Rio de Janeiro. 2016. Available from: http://www.ans.gov. br/images/stories/Materiais_para_pesquisa/Materiais_por_assunto/ web_final_livro_idosos.pdf. Accessed on: Mar 2, 2020.

Bacurau AGM, Ferraz RO, Donalisio MR, Francisco PMSB. Mortality due to cerebrovascular disease among the elderly and vaccination against influenza: São Paulo State, Brazil, 1980-2012. Cad Saude Publica. 2019;35(2):e00145117. 
Bocchi EA, Marcondes-Braga FG, Ayub-Ferreira SM, Rohde LE, Oliveira WA, Almeida DR, et al. III Diretriz Brasileira de Insuficiência Cardíaca Crônica. Arq Bras Cardiol. 2009:92(6 Suppl 1):1-71.

Bricks LF, Carvalhanas TRMP, Domingues CMAS, Pereira SF, Bellei NCJ. Influenza em pacientes com doenças cardíacas crônicas: o que há de novo? J Health Biol Sci. 2015;3(3):165-71.

Campagna AS, Dourado I, Duarte EC, Daufenbach LZ. Mortalidade por causas relacionadas à influenza em idosos no Brasil, 1992 a 2005. Epidemiol Serv Saúde. 2009;18(3):209-18.

Centers for Disease Control and Prevention. Morbidity and mortality weekly report. Prevention and control of influenza with vaccines: Recommendations of the Advisory Committee on Immunization Practices (ACIP) [Internet]. Vol. 59. 2010. Available from: https://www.cdc. gov/mmwr/pdf/rr/rr5908.pdf. Accessed on: Mar 2, 2020.

Cohen C, Simonsen L, Kang J, Miller M, Mcanerney J, Blumberg L, et al. Elevated influenza-related excess mortality in South African elderly individuals, 1998-2005. Clin Infect Dis. 2010;51(12):1998-2005.

Daufenbach LZ, Carmo EH, Duarte EC, Campagna AS, Teles CAS. Morbidade hospitalar por causas relacionadas à influenza em idosos no Brasil, 1992 a 2006. Epidemiol Serv Saúde. 2009;18(1):29-44.

Daufenbach LZ, Duarte EC, Carmo EH, Campagna AS, Santos CAST. Impacto da vacinação contra a influenza na morbidade hospitalar por causas relacionadas à influenza em idosos no Brasil. Epidemiol Serv Saúde. 2014;23(1):9-20.

Domingues CM, Oliveira WK; Brazilian Pandemic Influenza Vaccination Evaluation Team. Uptake of pandemic influenza (H1N1)-2009 vaccines in Brazil, 2010. Vaccine. 2012;30(22):4744-51.

Donalisio M, Ruiz T, Cordeiro R. Fatores associados à vacinação contra influenza em idosos em município do Sudeste do Brasil. Rev Saúde Pública. 2006;40(1):115-9.

Fleming D, Cross K, Pannell R. Influenza and its relationship to circulatory disorders. Epidemiol Infect. 2005;133(2):255-62.

Francisco PMSB, Barros MBA, Cordeiro MRD. Vacinação contra influenza em idosos: prevalência, fatores associados e motivos da não adesão em Campinas, São Paulo, Brasil. Cad Saude Pública. 2011;27(3):417-26.

GBD 2016 Lower Respiratory Infections Collaborators. Estimates of the global, regional, and national morbidity, mortality, and aetiologies of lower respiratory infections in 195 countries, 1990-2016: a systematic analysis for the Global Burden of Disease Study 2016. Lancet Infect Dis. 2018;18(11):1191-210.

GBD 2017 Influenza Collaborators. Mortality, morbidity, and hospitalisations due to influenza lower respiratory tract infections, 2017: an analysis for the Global Burden of Disease Study 2017. Lancet Respir Med. 2019;7(1):69-89.
Instituto de Estudos de Saúde Suplementar. Projeção das despesas assistenciais da Saúde Suplementar (2018-2030) [Internet]. 2019. Available from: https://www.iess.org.br/cms/rep/TD70_Projecao_ gastos_site20.pdf. Accessed on: Mar 2, Feb 14, 2020.

Ministério da Saúde (Brasil). Conselho Nacional de Saúde. Resolução n 510, de 07 de abril de 2016 [Internet]. 2016. Available from: https://conselho. saude.gov.br/resolucoes/2016/Res0510.pdf. Accessed on: Mar 2, 2020.

Ministério da Saúde (Brasil). Coordenação Geral do Programa Nacional de Imunizações, Departamento de Vigilância Epidemiológica, Secretaria de Vigilância em Saúde. Campanha nacional de vacinação contra a influenza: informe técnico. 19a edição [Internet]. 2017. Available from: http://pni.datasus.gov.br/sipni/03 032017 Informe_Cp_Influenza_final. pdf. Accessed on: Mar 2, 2020.

Ministério da Saúde (Brasil). Secretaria de Vigilância em Saúde. Boletim Epidemiológico. Monitoramento dos casos de influenza no Brasil, semanas epidemiológicas 1 a 32 de 2019. p. 1-9 [Internet]. 2019. Available from: https://portalarquivos2.saude.gov.br/images/pdf/2019/ setembro/05/BE-21-influenza-04set19.pdf. Accessed on: Mar 2, 2020.

Moura R, Andrade FB, Duarte YAO, Lebrão ML, Antunes JLF. Fatores associados à adesão à vacinação anti-influenza em idosos não institucionalizados, São Paulo, Brasil. Cad Saude Publica. 2015;31(10):2157-68.

Peasah SK, Azziz-Baumgartner E, Breese J, Meltzer MI, Widdowson MA. Influenza cost and cost-effectiveness studies globally - A review. Vaccine. 2013;31(46):5339-48.

Reed C, Chaves SS, Kirley PD, Emerson R, Aragon D, Hancock EB, et al. Estimating influenza disease burden from population-based surveillance data in the United States. PLoS One. 2015;10(3):e0118369.

Sato APS, Antunes JLF, Lima-Costa MFF, Bof de Andrade F. Influenza vaccine uptake among older adults in Brazil: Socioeconomic equality and the role of preventive policies and public services. J Infect Public Health. 2020;13(2):211-5.

Shrestha S, Foxman B, Berus J, Van Panhuis WG, Steiner C, Viboud C, et al. The role of influenza in the epidemiology of pneumonia. Sci Rep. 2015;5:1-13.

Smith SC, Benjamin EJ, Bonow RO, Braun LT, Creager MA, Franklin BA, et al. AHA/ACCF secondary prevention and risk reduction therapy for patients with coronary and other atherosclerotic vascular disease: 2011 update. A guideline from the American Heart Association and American College. Circulation. 2011;124(22):2458-73.

UNIDAS. Pesquisa Nacional UNIDAS 2017/2018 [Internet]. 2018. Available from: https://www.unidas.org.br/restrito/pesquisa-unidas. Accessed on: Mar 2, 2020.

World Health Organization. Vaccines against influenza. WHO position paper - November 2012. Wkly Epidemiol Rec. 2012;87(47):461-76. 